\title{
THE ASO REACTION - CLINICAL USES AND LABORATORY METHODS
}

\author{
Alexandra Gireada ${ }^{1}$, Nicolae Bacalbasa ${ }^{2}$, Irina Balescu ${ }^{3}$ \\ ',, Carol Davila“" Central Military Emergency Hospital, Bucharest, Romania \\ 2,"Carol Davila" University of Medicine and Pharmacy, Bucharest, Romania \\ ${ }^{3}$, ,Ponderas “ Hospital, Bucharest, Romania
}

\begin{abstract}
Abreviations:
ASO - Anti-streptolysin O

$\mathrm{CRP}$ - C-reactive protein

ADB - Anti-DNase B

ARF - Acute Rheumatic Fever

GAS infection - Group A streptococcal infection

SDS-PAGE - sodium dodecyl sulfate polyacrylamide gel electrophoresis

SLO - Streptolysin O
\end{abstract}

\section{INTRODUCTION}

ASO reaction is one of the most commonly used laboratory tests. This can be explained by the fact that the Group A Streptococcal infection is a frequent cause of angina and can cause severe complications and by the fact that $80-85 \%$ of the individuals having such an infection have also increased ASO titers. $(1,2)$

\section{ASO TITER - NORMAL VALUES}

The normal values of the ASO titer are less than 160 Todd units $/ \mathrm{ml}$ in adults, less than 50 Todd units/ml in children from six months to two years, less than 160 Todd units $/ \mathrm{ml}$ in children from two to four years, less than 170-330 Todd units/ml in children from five to 12 years. The concentrations in the newborn are similar to those of the mother. The anti DN-ase B titer is of less than 85 units in the adult population, less than 60 units in the preschool children and less than 170 units in children of school age. The results of the streptozyme test in healthy individuals must be of less than 100 streptozyme units $(3,4)$.

Epidemiological studies have demonstrated that the ASO titers are different in various populations and at different ages of the individuals. For example, in developed countries, the ASO concentrations are a consequence of the pharyngeal streptococcal infections and not of impetigo. Here, healthy people have low ASO concentrations in early childhood, a maximum of concentrations in children having 5-15 years of age, lower levels in late adolescence and early adulthood and constantly low levels in adult people (5).

Corresponding author:

Nicolae Bacalbasa, MD, Dimitrie Racovita Street no. 2, Bucharest, Romania

E-mail: nicolae_bacalbasa@yahoo.ro 


\section{THE SIGNIFICANCE OF INCREASED TITERS OF ASO VALUES}

Those populations that have an increased prevalence of impetigo are characterized by increased levels of the ASO titers, most frequently in children. An explanation is represented by the fact that most of them were tested after a recent streptococcal infection. The ASO titer depends also on the moment of determination. It rises immediately after infection, has a maximum at 3-5 weeks and declines since the $8^{\text {th }}$ week after the infection. The ASO titer also depends on the type of infection, being higher in the pharyngeal infection than in skin infection. The ADB titer has a similar dynamic, having a peak concentration at 6-8 weeks and starting to decline after 12 weeks. The ADB titer is considerably raised in both pharyngeal and skin infections $(5,6)$.

The association of ASO titers, CRP values can distinguish between different acute rheumatic conditions. CRP values are also elevated, $>1.2 \mathrm{mg} / \mathrm{dL}$ in about $80 \%$ of the patients with a Group A Streptococcal infection. The presence in the serum of Bacillus cereus and Pseudomonas can lead to falsely elevated ASO levels. False positive results can be found also in patients with liver diseases who have increased concentrations of serum beta-lipoprotein (2).

It is recommended that the test be done twice, at an interval of two weeks, in order to see the dynamics of the concentrations. The ASO antibodies are rising during the first month after infection and have the highest concentration at 3-5 weeks after the onset of the infection. Then, the titer is decreasing for several months to undetectable concentrations (1). A recent infection is suggested by the presence of an at least double concentration at a second determination (7). In the presence of the complications or of the re-infection, the concentrations do not become normal. The ASO titers can't predict the disease severity or the probability of the complications (1).

The main utility of the ASO titer and of antiDNase B is the etiological diagnosis of glomerulonephritis and of rheumatic fever. The signs and symptoms characteristic for glomerulonephritis are: fatigue, low urinary volume with the presence of blood in the urine, increased blood pressure, edema, rash and joint pain. The signs and symptoms characteristic for rheumatic fever are: fever, edema and pain affecting at least one joint and moving from one joint to another, subcutaneous painless nodules, signs of Sydenham's chorea, skin rash, signs of cardiac involvement like palpitations or chest pain (1).

In rheumatic fever there are used two types of laboratory investigations: the acute phase reactants and the tests that can prove a previous streptococcal infection. None of them can be used as a diagnostic test (8).

The main acute phase reactants used are the erythrocyte sedimentation rate, the C-reactive protein and the serum mucoproteins. They are good indicators of disease activity. A preceding streptococcal infection can be diagnosed by bacteriologic and immunologic methods.

There are considerable individual variations in the time necessary for the ASO concentrations to return to normal. The factors that influence the rate of decline are steroid and antibiotic treatment (accelerator), the persistence of the organism, the presence or the absence of tonsils (prolonging the normalization period), reinfection (characterized by a sustained or permanently increasing ASO level). There are also individual variations in the highest antibody titer, depending on the severity of the streptococcal infection or on the serologic type of the group A streptococcus (there are more than 40 serologic types, classified upon the surface M proteins). Very high antibody titers were seen in patients who developed rheumatic fever. The amplitude of the antistreptococcal response depends also on the number of previous streptococcal infections, the patient's age and the residual antibody level at the moment of reinfection. The ASO titers are generally higher in older children or adults than in infants (1).

There are several problems that appear in the clinical practice. One of them is the ASO determination when a complication appears, when the concentration is maximum, so we can't see the rise at a second determination. To correctly interpret the titer obtained at the moment we suspect the complication, we have to compare it with the baseline titer of the patient, not with the average concentration in the population. The mean ASO titers in different populations depend on the age, the geographic region, the economical state, the season, the year or other variables. There are patients who present with normal ASO concentrations at the time when chorea begins $(8,9)$ (this is the case of patients with isolated chorea, which appears at 1-6 months after the streptococcal infection) (9). This is due to a long latent period since the acute streptococcal infection. There are also patients in whom the ASO concentrations continue to rise even after the beginning of the acute rheumatic fever. About $20 \%$ of 
the patients with streptococcal infections don't have an increased ASO concentration. Literature data also sustain that about $20 \%$ of the patients with acute rheumatic fever have low ASO concentrations (lower than 250 units), leading to the conclusion that the normal ASO titer can't exclude the diagnosis of acute rheumatic fever. In the category of patients mentioned above, the determination of other antibodies with different dynamics of the concentrations can be useful: antistreptokinase, antihyaluronidase, antidiphosphopydrine-nucleotidase (anfi-DPNase), antidesoxvribonuclease B (antiDNAse B) (8).

The antibodies present in the group A streptococcal infections can be detected by means of the following laboratory tests: the antistreptolysin $\mathrm{O}$ titer (ASO), the antideoxyribonuclease-B titer (anti-DNase-B) and the streptozyme test. The administration of antibiotics or steroids can decrease the antibody titers. The concomitant use of ASO and anti-DNase-B antibodies can retrospectively detect about $95 \%$ of the cases of streptococcal infections. This sensitivity is higher than that of ADB titer alone and this one has a higher sensitivity than the ASO titer. However, because the results of the antiDNase-B testing are variable, the American Heart Association recommends the use of the ASO titers. The streptozyme test detects the antibodies developed against the streptococcal antigens NDase, DNase, streptokinase, streptolysin $\mathrm{O}$ and hyaluronidase. The advantages of this test are that it can easily and rapidly detect several antibodies, without the interference of false-positive reactions. The disadvantages of this test are that it can't determine each single antibody and that the sensitivity in children is lower than in adult population (3).

In patients with ARF, the ASO titers are higher than in patients with other conditions. This means that the ASO titers can be used to differentiate the ARF associated with polyarthritis from other diseases like the Juvenile Idiopathic Arthritis (JIA), the systemic lupus erythematosus or the acute lymphoblastic leukemia. This is very important since there are literature data that sustain that the atypical presentation and evolution of the polyarthritis are present in about $47 \%$ of the ARF cases (9).

A study realized at São Paulo in 2001 included 100 patients diagnosed with rheumatic fever, 45 patients with recurrent oropharyngeal infections and 23 patients with juvenile idiopathic arthritis. The ASO levels were determined on a 5 year period starting with the moment of the diagnosis by means of a latex agglutination nephelometric assay, a method which gives similar results to the hemoly- sis inhibition assays ( $1 \mathrm{IU}=1.04 \mathrm{U}$ Todd). The results showed that the patients with recurrent oropharyngeal infections had significantly lower levels of the ASO titers than the patients with ARF, isolated chorea or juvenile idiopathic arthritis $(p<0.05)$ The patients diagnosed with ARF had the highest ASO titers $(p<0.0025)$ (9).

A possible explanation for the low ASO titer specificity resides in the presence of recent streptococcal infections in patients with arthritis caused by diseases other than ARF (9).

A possible explanation for the ASO sensitivity is that there are cases of skin infections or renal complications without an elevated ASO titer (2).

The antistreptolysin $\mathrm{O}$ titer determination can be used to diagnose infections caused by the $\beta$-hemolytic group $\mathrm{A}, \mathrm{C}$ and $\mathrm{G}$ streptococci. In adult patients, at a cut-off of $400 \mathrm{U} / \mathrm{ml}$, it has sensitivity and a specificity of $66 \%$ and $82 \%$ for the detection of the S. pyogenes infections of the upper respiratory tract (Gray et al.). ASO can be extremely useful to diagnose these infections in patients already treated with empirical antimicrobial agents. This situation can occur in patients with orthopedic infections, which are generally caused by staphylococci (two-thirds of the cases), but can be caused also by streptococci (7-9\% of the cases) (4).

A study realized in 2008 at the University Hospital of Geneva included adult patients who had orthopedic-related infections diagnosed by positive microbiological cultures obtained by means of aspiration or biopsy. The ASO titers were determined by means of a semi-quantitative test which had a cut-off value of $200 \mathrm{U} / \mathrm{ml}$. The principle of the test was based on the hemolysis of rabbit erythrocytes by the streptolysin $\mathrm{O}$ obtained from Streptococcus pyogenes culture, hemolysis that can be neutralized by the antibodies against streptolysin $\mathrm{O}$ found in the patient's serum (4).

The concentration of the C-reactive protein was measured several times in each patient. The maximum values were situated in the interval 5-499 $\mathrm{mg} / \mathrm{l}$, having an average of $117.4 \mathrm{mg} / \mathrm{l}$. The ASO titer was also determined several times, a first time at 13 days after the infection onset and a last time at 16 days. The average concentration in the ASO negative patients was $100 \mathrm{U} / \mathrm{ml}$. The positive values were situated in the interval $300-800 \mathrm{U} / \mathrm{ml}$, with an average concentration of $600 \mathrm{U} / \mathrm{ml}$. These patients were diagnosed with infections with $\beta$ - hemolytic group A, C and G streptococci. There were not false-positive results. There was only one negative result, because of the short interval of about three days between the onset of the infection and 
the ASO determination. The conclusion of the study was that in orthopedic patients with negative cultures, the presence of positive ASO titers can lead to the administration of narrow spectrum antibiotics, such as penicilins (4).

Increased concentrations of antistreptolysine $\mathrm{O}$ antibodies can be also found in patients with noninfectious arthritis and sacroiliac joint affections, according to literature data. These patients usually have a genetic susceptibility (4).

Since the description of the first method for the determination of the ASO titer, realized by Todd, several modified methods have been proposed, in order to make it more practical. One of them, discovered by Ricci and his colleagues, used various quantities of oxidized Streptolysin O (SO), a reducing agent (2-mercaptoethanol) and whole blood samples. It was a manual microtechnique (6).

In 1984, A. Ricci described an automated method which determines the kinetics of the reaction used by the haemolytic tests. The anti-Streptolysin $\mathrm{O}$ titer is then measured without the dilution of the sample which is represented by the whole blood. The reaction starts by the addition of a reducing agent (L-cysteine) to the oxidized Streptolysin O. The initial rate of the reaction and the ASO titer of the sample are in a relation of inverse proportionality. All these procedures were effectuated by means of an automatic instrument. Between the results obtained and those of the classic haemolytic method there was a correlation which could be described by the following equation: $\mathrm{y}=1.06 \mathrm{x}-13(\mathrm{r}=0.974)$ (6).

Both methods were used in order to test 591 samples. Although 48 of them had hematocrit values of $30-40 \%$ (with a higher initial reaction rate and falsely lower titers), the difference between the results obtained by the two methods was always less than $100 \mathrm{IU}$. This can be explained by the fact that, in these 48 samples, there is an increased plasma volume, an increased number of antibodies which leads to increased ASO titers (6).

The first laboratory test utilized for the ASO determination was the haemolysin method. Today, one of the most frequently used determination methods is latex agglutination, which uses particles that are pre-sensitised with streptolysin O. A study which compared the two methods was realized by G.D.W. Curtis et al. in 1988 by means of the serum samples taken from 428 individuals. They were stored at $4{ }^{\circ} \mathrm{C}$ for a week and at $-20^{\circ} \mathrm{C}$ for up to one year. Using a cut-off value of $200 \mathrm{IU} / \mathrm{ml}$, he obtained a sensitivity, specificity, positive predictive value and negative predictive value of $83.6 \%$,
$93.3 \%, 86.5 \%$ and $91.6 \%$ for the latex agglutination method. As compared to the haemolysin test, the latex test was more convenient, easy to realize, giving also the possibility of testing a reduced number of samples without an economic disadvantage. The haemolysin test required more time to perform, a higher price or the batching of sera because the reduced streptolysin $\mathrm{O}$, once reconstituted, was available only for six hours (10).

The authors also studied the influence of different speeds of the mechanical rotator and of the execution of the manual rotation by different persons on the results obtained. They found a correlation between a high rotation speed and a high ASO titer, which lead to the necessity of having a standard method. The reading phase was subjective in both methods. At low titers, the results obtained by means of the two methods were significantly different. Fewer differences between the haemolysin inhibition method and the latex agglutination method were obtained using a nephelometer for the reading of the results from the latex test (suggesting an increased sensitivity) and these were encountered at high titers. In conclusion, the authors highlighted the advantages of the latex agglutination method, which make it affordable even by the smallest laboratories (10).

A study realized by Ota $\mathrm{H}$ investigated the presence of pseudo-positive reactions given by the latex agglutination method in healthy adults. It included the serum samples from 159 healthy individuals and 8 serum samples from patients with documented GAS infection. $15 \%$ of the healthy individuals with ages lower than 30 had ASO levels > $200 \mathrm{IU} / \mathrm{ml}$. The individuals with ages higher than 30 years had ASO titers $<200 \mathrm{IU} / \mathrm{ml}$. When tested with SDS-PAGE, the SLO utilized by the latex method had more than three protein bands, but only one was considered SLO protein because it had a molecular weight of $64 \mathrm{kD}$. Most of the ASO antibodies in the healthy adults included in this study were of the IgG class. Some of them were of the IgG class. The antibodies present in the patients' serum combined with the $64-\mathrm{kD}$ protein, which was the SLO and the antibodies present in the serum of the healthy individuals combined with different proteins during an immunoblotting analysis. The conclusion of the study was that some of the healthy adults with elevated ASO titers had other antibodies than ASO in their serum and thus pseudo-positive results on the ASO determination, the solution to this problem being the use of more specific proteins by the commercial kits (11). 
A study realized in 1995 by Pacifico L et al. compared a new nephelometric method with the old hemolytic inhibition assay. It included 330 serum samples from children without the evidence of a recent group A streptococcal infection, 134 serum samples from patients diagnosed with acute and convalescent GAS infection on the basis of microbiological cultures and 50 serum samples from control subjects. The results demonstrated a good correlation between the two methods ( $\mathrm{r}=0.88$ ), but the automated nephelometric method had a higher sensitivity for the determination of significant ASO antibody rises. Another advantage of this method is that it has an easier way to perform (12).

A study realized by Freyaldenhoven BS et al. compared a method using recombinant antigens on the Beckman Coulter IMMAGE System with a nephelometric method based on conventional antigens. It included 114 patients not necessarily diagnosed with GAS infection on the basis of the mi-

\section{$\overline{\text { REFERENCES }}$}

1. http://labtestsonline.org/understanding/analytes/aso/tab/test/

2. http://scantibodies.com/aso/

3. http://medical-dictionary.thefreedictionary.com/ Streptococcal+Antibody+Tests

4. Uckay I., Ferry T., Stern R. et al. Use of serum antistreptolysin O titers in the microbial diagnosis of orthopedic infections. Int J Infect Dis. 2009; 13(4):421-424.

5. Steer A.C., Vidmar S., Ritika R. et al. Normal ranges of streptococcal antibody titers are similar whether streptococci are endemic to the setting or not. Clin Vaccine Immunol. 2009; 16(2):172-175.

6. Ricci A., Arezzini C., Cocola F., Meiattini F. Automated determination of anti-streptolysin $\mathrm{O}$ antibodies by a kinetic hemolytic method. J Clin Microbiol. 1984; 20(6):1065-1067.

7. Teco Diagnostics - Antistreptolysin O (ASO) Reagent Set, A Latex Slide Test

8. Wannamaker L.W., Ayoub E.M. Antibody titers in acute rheumatic fever. Circulation. 1960; 21:598-614.

9. Machado C.S., Ortiz K., Martins A.L. et al. Antistreptolysin O titer profile in acute rheumatic fever diagnosis. J Pediatr (Rio J). 2001; 77(2):105-111. crobiological cultures. There was a good correlation between the results obtained by the two methods. The combined determination of both antistreptolysin $\mathrm{O}$ and anti DN-ase B antibodies had a higher sensitivity than the determination of a single antibody alone. The newly proposed method using recombinant antigens had the advantage of an easier handling of the tests (13).

\section{CONCLUSIONS}

ASO reaction is an important laboratory test which can determine the status of Group A Streptococcal infection; in specific cases association of other laboratory tests such as antistreptokinase, antihyaluronidase, antidiphosphopydrine-nucleotidase (anfi-DPNase), antidesoxyribonuclease B (antiDNAse B) might be needed in order to provide a more complete evaluation of these patients.

10. Curtis G.D., Kraak W.A., Mitchell R.G. Comparison of latex and haemolysin tests for determination of anti-streptolysin O (ASO) antibodies. J Clin Pathol. 1988; 41(12):1331-1333.

11. Ota H., Sato A., Matsumoto H. et al. Immunological analysis of pseudo-positive reaction in healthy adults at anti-streptolysin $\mathrm{O}$ measurement by latex agglutination method. Rinsho Byori. 2005; 53(4):279-283.

12. Pacifico L., Mancuso G., Properzi E. et al. Comparison of nephelometric and hemolytic techniques for determination of antistreptolysin O antibodies. Am J Clin Pathol. 1995; 103(4):396399.13. Freyaldenhoven B.S., Moos G., Hinzmann R.D., Lutticken R.

13. Evaluation of recombinant antigens for the measurement of antibodies to streptolysin O and streptococcal DNase B on the Beckman Coulter IMMAGE system and comparison with a method employing conventional antigens. Clin Lab. 2004; 50(11-12):667-673. 